
\title{
.
}

\section{BMP15 Prevents Cumulus Cell Apoptosis Through CCL2 and FBN1 in Porcine Ovaries}

\author{
Bo Zhaia Huiyu Liu ${ }^{a}$ Xu Lib Lisheng Daia Yan Gao Changhong Lia Lianjiang \\ Zhang $^{a}$ Yu Ding ${ }^{a}$ Xianfeng Yu ${ }^{a}$ Jiabao Zhang ${ }^{a}$ \\ aCollege of Animal Science, Jilin University, Changchun 130062, China; bJilin Academy of Agricultural \\ Sciences, Changchun136100, China
}

\section{Key Words}

BMP15 • Cumulus cell • Apoptosis • CCL2 • FBN1

\begin{abstract}
Background: Bone morphogenetic protein-15 (BMP15) is a maternal gene necessary for mammalian reproduction. BMP15 expression increased in oocytes accompanied by follicle growth and development. The function and regulation mechanism of BMP15 in porcine cumulus cell apoptosis process is still unclear now. Methods: In this study, flow cytometry (FCM) was used to analyze the effects of BMP15 with different concentrations to cumulus cell apoptosis. High-throughput sequencing technology was carried out to screen regulatory genes linked closely with BMP15. In order to confirm the function of (MCP-1)/CCL2 and FBN1 in cumulus cell apoptosis, RNA interference (RNAi) method was used to inhibit the expression of (MCP-1)/CCL2 and FBN1. Apoptosis and proliferation of cumulus cell treated with siRNA transfection technology were measured by FCM, 3-(4,5-dimethylthiazol-2-yl)-2,5-diphenyltetrazolium bromide, quantitative real time-PCR (RT-qPCR) and western blotting. Results: The results showed that the apoptosis levels of cumulus cell treated by BMP15 decreased significantly in a dose-dependent manner. The expression of related genes protein 1 (MCP-1)/ CCL2 and fibrillin1 (FBN1) were both regulated by BMP15. After transfection, the proliferation of porcine cumulus cells increased significantly and apoptosis of cumulus cells was prevented while FBN1 was silenced after BMP15 treatment. The proliferation of cumulus cells decreased significantly and apoptosis rate of cumulus cells increased significantly while CCL2 was silenced. Conclusion: The results obtained in this study firstly demonstrated that CCL2 and FBN1 are important regulatory factors of BMP15 in preventing cumulus cell apoptosis in porcine ovaries.
\end{abstract}

J-B Zhang

and $\mathrm{X}-\mathrm{F} \mathrm{Yu}$
College of Animal Science, Jilin University, Changchun 130062 (China)

E-Mail zjb515@163.com

and College of Animal Science, Jilin University, Changchun 130062 (China)

E-Mail xianfeng79@126.com 
Zhai et al.: Effects of BMP15 on Porcine Cumulus Cell Apoptosis

\section{Introduction}

Most follicles in mammalian ovaries showed degradation and atresia in different development stages. It is shown from predecessors' studies that except $1 \%$ of follicles develop to maturity and ovulation, the remaining follicles undergo atresia [1]. Many researches on a variety of animals show that mammalian follicular atresia is mainly caused by the apoptosis of granulosa cells [2]. Apoptosis is basic physiological mechanisms which make bodies maintain their stability. Apoptosis is an active cellular suicide process encoded by genes during the organisms' growth, differentiation, development and pathological processes. Initiation of the cells apoptosis may be triggered by receiving cytotoxic signals or by receiving survival or growth signals [3]. Studies on granulosa cell apoptosis will help to a better understanding of the underlying mechanisms in follicular atresia. Although apoptosis is an autonomous process which regulated by a cell's own genes, many extracellular factors also has influence to apoptosis. In some cases, the extracellular factors were very critical to apoptosis. As well as the pathological processes, during organisms' growth and development, extracellular factors influence the intracellular regulatory genes mediating cell survival via a variety of signal transduction pathways which determine the particular cell survival or death. Apoptosis is different from necrosis. Once a series of mRNAs were transcripted and translated after apoptosis-related genes have been activated, the cells will soon undergo apoptosis. In contrast, apoptosis might not occur if synthesis of RNA or protein be inhibited. Granulosa cells are called ovary somatic cells. The regulation mechanism of apoptosis in granulosa cell is similar to normal cells. For example, granulosa cell apoptosis is regulated by apoptotic genes such as Bcl2, Bax and p53. In addition, granulosa cell apoptosis has unique regulatory factors and modes due to the unique physiological environment and developmental processes.

Bone morphogenetic protein-15 (BMP15) which is also known as growth differentiation factor 9B (GDF9B), belongs to the transforming growth factor- $\beta$ (TGF- $\beta$ ) superfamily and secreted by oocytes. BMP15 mRNA is uniquely located in oocytes in sheep ovaries [4]. BMP15 expression occurred in all human, mouse and rat oocytes in the early stages of follicular development. The expression level of BMP15 in the dominant ovarian follicles of the mouse is higher than that in the atretic follicles [5]. BMP15 stimulates the proliferation of granulosa cells [6] and is involved in the regulation of regulatory factors such as follistatin [7], the kit ligand (KL) [8], Gremlin [9] and inhibits FSH receptor expression. BMP15 and the KL form a novel gamete-somatic cell negative feedback loop that is functionally linked to granulosa cell proliferation [8]. BMP15 is also involved in the regulation of cumulus cell apoptosis. In bovine ovaries, removal of the oocyte from cumulus-oocyte complexes (COC) induces cumulus cell apoptosis. The induction of cumulus cell apoptosis can be prevented by treatment with BMP15 [10]. Thus, BMP15 may contribute to decreasing the apoptotic rate within COC until the point of ovulation. Comparing the unique structure of BMP15 with other TGF- $\beta$ superfamily members, it is possible that BMP15 uses specific receptors and signaling to regulate apoptosis or other biological processes. Currently, nothing is known about the mechanism which BMP15 modulates apoptosis in granulosa cells.

Many studies have investigated granulosa cell apoptosis in pigs, and many factors have been reported to take part in this process, such as tumor necrosis factor $\alpha$ (TNF $\alpha$ ) and its receptor [11], factor associated suicide (FAS)/FAS ligand (FASL) [12], and TNF-related apoptosis-inducing ligand (TRAIL) and its receptor [13]. In this study, the expression of mRNAs was evaluated in porcine cumulus cells after BMP15 treatment using a next-generation highthroughput sequencing technology. (Follistatin produced by mural granulosa cells (MGCs) and present in follicular fluid may limit the anti-apoptotic effects of BMP15, which is secreted by oocytes specifically in the COC microenvironment [10]. Thus, the porcine cumulus cells, and not the MGCs, were employed in this study). Dozens of differentially expressed mRNAs were identified, and the functions of these mRNAs were predicted. Among these genes, apoptosisrelated genes were screened out and it is found that BMP15 treatment significantly affected the expression of two apoptosis-related genes, chemokine (C-C motif) ligand 2 (CCL2) and 
fibrillin 1 (FBN1). RNA interference (RNAi) technology was carried out in this study, the results showed that CCL2 and FBN1 were closely related to porcine cumulus cell apoptosis and FBN1 induced porcine cumulus cell apoptosis and CCL2 protected porcine cumulus cells from apoptosis in vitro with BMP15 treatment,. This is the first evidence that CCL2 and FBN1 are important factors regulating the apoptosis process which BMP15 protects porcine cumulus cells. The results obtained will provide a theoretical basis for revealing the mechanism underlying porcine granulosa cell apoptosis in further studies.

\section{Materials and Methods}

\section{Cumulus cell separation and culture}

Unless otherwise specified, all chemicals and reagents were purchased from Sigma (St Louis, MO). Porcine ovaries were collected from local abattoirs and transported to the laboratory in warm saline $\left(35-37^{\circ} \mathrm{C}\right)$. Follicular fluid was collected from antral follicles (3-8 $\mathrm{mm}$ diameter). COC were mechanically aspirated from antral follicles using an 18-gauge needle and a $10 \mathrm{ml}$ syringe containing $2 \mathrm{ml}$ aspiration media (Hepes-buffered DMEM-F12). Intact COC with compact cumulus vestments greater than five cell layers and evenly pigmented cytoplasm were selected under a dissecting microscope and washed 2-3 times in Hepes-buffered DMEM-F12, and we dissociated the cumulus cells by incubation with hyaluronidase $(0.1 \%)$ for three minutes. The cumulus cells were then washed 2-3 times with DMEM-F12. Suspension cells were treated in the cell culture plate, and DMEM/F12 (penicillin \&streptomycin and 10\%fetal calf serum) with $0 \mathrm{ng} / \mathrm{ml}, 25 \mathrm{ng} / \mathrm{ml}, 50 \mathrm{ng} / \mathrm{ml}, 100 \mathrm{ng} / \mathrm{ml}$ or $200 \mathrm{ng} / \mathrm{ml}$ recombinant human BMP15 (R\&D systems) was added to the plate and incubated at $38.5^{\circ} \mathrm{C}$ with $5 \% \mathrm{CO}_{2}$ in humidified air for 96 hours (the logarithmic phase of cells).

Flow cytometric analysis of apoptotic cells

Apoptotic cells were differentiated from viable or necrotic cells by the combined application of annexinV-FLUOS and propidium iodide (PI) (Roche, Germany). The three parallel samples were washed twice and adjusted to a concentration of $1 \times 10^{5} \mathrm{cells} / \mathrm{mL}$ with $4^{\circ} \mathrm{C}$ PBS. In total, $100 \mu \mathrm{L}$ of cell suspension was added to each labeled Falcon tube $(12 \mathrm{~mm} \times 75 \mathrm{~mm}$, polystyrene round-bottom). Next, $10 \mu \mathrm{L}$ of annexin-V-FLUOS and $10 \mu \mathrm{L} \mathrm{PI}(20 \mu \mathrm{g} / \mathrm{mL})$ were added into the tube and incubated for at least $20 \mathrm{~min}$ at room temperature in the dark. Then, $400 \mu \mathrm{L}$ of PBS binding buffer was added to each tube without washing, and the sample was analyzed using FCM analysis (Roche, Germany) immediately (within $30 \mathrm{~min}$ ). This assay was performed in triplicate.

\section{Library construction and sequencing}

Total RNA was extracted from the cumulus cells treated with $0 \mathrm{ng} / \mathrm{ml}$ and $100 \mathrm{ng} / \mathrm{ml}$ BMP15 using standard protocols (Trizol). The RNA was treated with DNase to remove potential genomic DNA contamination according to the manufacturer's protocols. RNA integrity and concentration were evaluated using the Agilent 2100 Bioanalyzer (Agilent Technologies). For RNA library construction and deep sequencing, equal quantities of RNA from three parallel N-BMP15 cumulus cells samples were pooled, and RNA samples from the three parallel BMP15 treatment cumulus cells sample were pooled. Approximately 8 $\mu \mathrm{g}$ of RNA representing each group were submitted to Solexa (now Illumina Inc.) for sequencing. Sequence tag preparation was performed using Illumina's Digital Gene Expression Tag Profiling Kit according to the manufacturer's protocol. In brief, mRNA was isolated from $8 \mu$ g total RNA by binding the mRNA to a magnetic oligo bead. First- and second-strand CDNA were synthesized while the mRNA was attached to the beads. The double stranded cDNAs were separated with NlaIII to wash away all fragments other than the 3'CATG fragment attached to the oligo bead. Then, GEX NlaIII Adapter 1 was bound at the site of NlaIII cleavage. GEX NlaIII Adapter 1 also contains the sequence for the restriction enzyme MmeI, so we applied the restriction enzyme MmeI to create the $17 \mathrm{bp}$ tag. The GEX Adapter 2 was bound at the site of MmeI cleavage. A PCR with 12 cycles was performed with two primers that anneal to the ends of the adapters to enrich the adapter-bound cDNA construct. The resulting $85 \mathrm{bp}$ fragments were purified from a $6 \%$ Novex TBE PAGE gel. Subsequently, the purified cDNA tags were sequenced on the Illumina Cluster Station and Genome Analyzer. Finally, image recognition and base calling were performed using the Illumina Pipeline. 
Table 1. Primer sequences used for RT-PCR

\begin{tabular}{|c|c|c|c|c|}
\hline Gene & Accession No & Primer sequence $5^{\prime}-3^{\prime}$ & $\begin{array}{l}\text { Product size } \\
\text { (bp) }\end{array}$ & $\begin{array}{c}\text { Annealing } \\
\text { temperature }\left({ }^{\circ} \mathrm{C}\right) \\
\end{array}$ \\
\hline$\beta$-Actin & NC_010445 & $\begin{array}{l}\text { F-CGTGACATTAAGGAGAAGCTGTGC } \\
\text { R-CTCAGGAGGAGCAATGATCTTGAT }\end{array}$ & 163 & 61 \\
\hline IGF1R & NM_214172 & $\begin{array}{l}\text { F- TCAACGCCAATAAGTTTGTCC } \\
\text { R- CCTCCCTTGCGGTAATAGTCT }\end{array}$ & 133 & 60 \\
\hline CKAP2 & XM_003130956 & $\begin{array}{l}\text { F- ATGTGACGACTGCCAAGAAAT } \\
\text { R- ATCCTACTGATGTCCCGTTTG }\end{array}$ & 130 & 59 \\
\hline TAK1B & EU399819 & $\begin{array}{l}\text { F- AGCCTATTCCAAGCCTAAACG } \\
\text { R- GGATGACCTACTGCTCACCTG }\end{array}$ & 166 & 60 \\
\hline BMPR2 & XM_003133596 & $\begin{array}{l}\text { F- GCAGCAAGCACAAATCAAACT } \\
\text { R- ACATACTGGGTAGTGGCAGCA }\end{array}$ & 157 & 60 \\
\hline TOPOII & NM_213884 & $\begin{array}{l}\text { F-TGTGGAAACAGCCAGTAGAGAA } \\
\text { R-TAGACAAATCAGGGTGGAAGGT }\end{array}$ & 140 & 60 \\
\hline TRIO & XM_003133838 & $\begin{array}{l}\text { F- TCAGCCATCTCCAGTAACAAGA } \\
\text { R- GTCCTCCGTGTCAAAGTCATTC }\end{array}$ & 240 & 60 \\
\hline CAV2 & NM_001123091 & $\begin{array}{l}\text { F- TTCATGGACGACGACTCCTAC } \\
\text { R- TGCTATCACATCCTCGAATCC }\end{array}$ & 138 & 60 \\
\hline Alpha-1c & XM_003126113 & $\begin{array}{l}\text { F-CTTCAGGGCTTCTTGGTTTTC } \\
\text { R-TGGTGAGGATGGAGTTGTAGG }\end{array}$ & 178 & 60 \\
\hline L1CAM & XM_003132328 & $\begin{array}{l}\text { F-ATCATCCTCCTGCTTCTGGTC } \\
\text { R-TGGGTGTCCTCCTTATCCTTC }\end{array}$ & 92 & 60 \\
\hline SFRP2 & XM_003129134 & $\begin{array}{l}\text { F-CAGCCCGACTTCTCCTACAA } \\
\text { R-ATGGTTTCGTCCAGGTCATC }\end{array}$ & 245 & 60 \\
\hline MMP14 & NM_214239 & $\begin{array}{l}\text { F-CAAGCCCTCTGTTCCTGATAA } \\
\text { R-TCTTTCCTCTCGTAGGCAGTG }\end{array}$ & 225 & 59 \\
\hline
\end{tabular}

\section{RT-QPCR of gene expression}

To verify the DEG results, RT-PCR analysis was carried out. The RNA samples used for RT-PCR assays were independent to RNA extractions from biological replicates, the same as for the Illumina experiments. RT-PCR was performed on the Mastercycler ${ }^{\circledR}$ ep realplex (Eppendorf, Germany) with SYBR-Green detection (SYBR PrimeScript RT-PCR Kit, TaKaRa Biotechnology Co., Ltd.), according to the manufacturer's instruction. Primer sequences, the size of amplification products and the GenBank accession numbers are shown in Table 1.The total volume of 20ul RT-PCR reaction mixture contained 10ul of SYBR ${ }^{\circledR}$ Green Real-time PCR Master Mix, $2 \mathrm{ul}$ of cDNA and 0.5uM each of forward and reverse primers. The program used for all genes consisted of a denaturing cycle of $120 \mathrm{~s}$ at $95^{\circ} \mathrm{C}, 40$ cycles of PCR $\left(94^{\circ} \mathrm{C}\right.$ for $30 \mathrm{~s}, 59^{\circ} \mathrm{C}-61^{\circ} \mathrm{C}$ for $30 \mathrm{~s}$ and $72^{\circ} \mathrm{C}$ for $60 \mathrm{~s}$ ), a melting cycle consisting of $95^{\circ} \mathrm{C}$ for $30 \mathrm{~s}, 72^{\circ} \mathrm{C}$ for $5 \mathrm{~min}$ and a step cycle starting at $65^{\circ} \mathrm{C}$ with a $0.2^{\circ} \mathrm{C} / \mathrm{s}$ transition rate to $95^{\circ} \mathrm{C}$. The specificity of the RT-PCR product was confirmed by melting curve analysis. Each cDNA was analyzed in triplicate, after which the average threshold cycle (Ct) was calculated in each sample. The relative expression levels were calculated with the $2^{-\Delta \Delta C t}$ method. The results were normalized to the expression level of $\beta$-actin and relative to the N-BMP15 sample.

\section{Design and transfection of siRNA}

The complementary DNA (cDNA) sequence of porcine CCL2 and FBN1 (GenBank accession No. NC_010454 and NC_010443) was examined using Invitrogen's web-based siRNA design software (https://rnaidesigner.invitrogen.com/ rnaiexpress/) to select appropriate siRNA target sites. A pair of oligonucleotides corresponding to CCL2 and FBN1 cDNA was designed. A BLAST (basic local align-ment search tool) search of these sequences confirmed their specificity to CCL2 and FBN1 only. In addition, a nonsense sequence with no similar match to any known sequence was used as control. The CCL2 and FBN1 small RNA interference (RNAi) was named siRNA-CCL2 and siRNA-FBN1, and the nonsense sequence to CCL2 and FBN1 was named negative control siRNA (NC-siRNA). Cumulus cells cultures were transfected with siRNA-CCL2 and siRNA-FBN1 or NC-siRNA using Lipofectamine ${ }^{\mathrm{TM}}$ RNAiMAX Reagent (Shanghai Invitrogen Biotech-nology Co., Ltd.). Briefly, RNAi duplex, Lipofec-tamine ${ }^{\mathrm{TM}}$ RNAiMAX, and $250 \mu \mathrm{l}$ DMEM/ F12 medium were combined and incubated for $20 \mathrm{~min}$ at room temperature. The transfection complex was added to the cells, and incubated for $48 \mathrm{~h}$ at $37^{\circ} \mathrm{C}$.

\section{Measurement of apoptosis related genes expression by Western blot}

Total proteins from cumulus cells were extracted in a cell lysis buffer for Western and immunol precipitation (Beyotime, China) containing $10 \mathrm{mmol} / \mathrm{L}$ phenyl-methyl sulfonylfluoride (PMSF; Beyotime, 

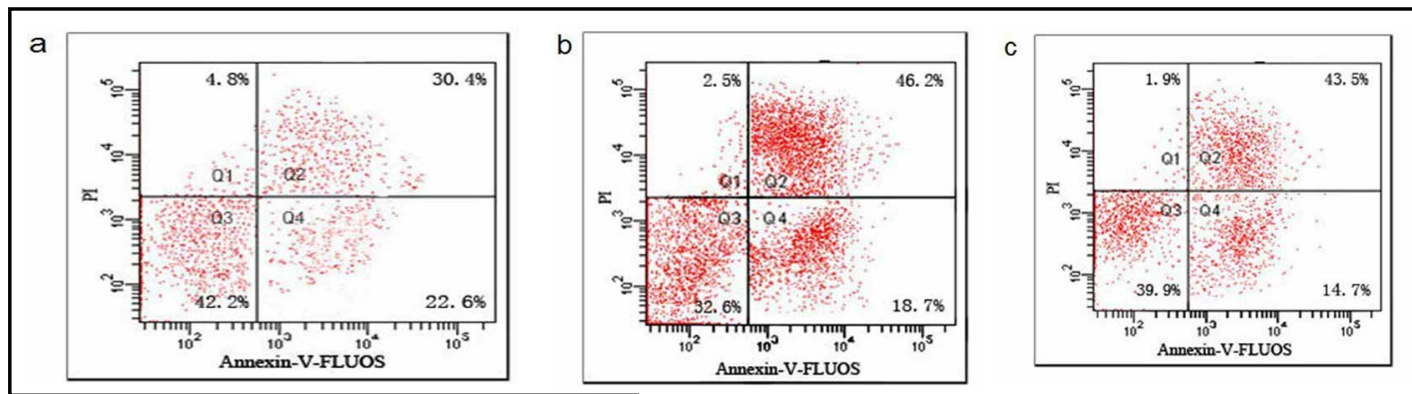

Fig. 1. BMP15 reduces apoptosis rate in cumulus cells. Cumulus cells were treated with 0-200 ng/ml BMP15. Apoptosis was determined by annexin V-FLUOS intensity and mitochondrial transmembrane potential (DWm) depolarization ratio with flow cytometry on the FLUOS or phyco-erythrin (PE)-Texas Red channel. a,b,c,d,e representative images of flow cytometry showing annexin V-FLUOS and DWm depolarization ratio in cumulus cells. a,0ng/ml; b,25ng/ml; c,50ng/ml; $\mathrm{d}, 100 \mathrm{ng} / \mathrm{ml} ; \mathrm{e}, 200 \mathrm{ng} / \mathrm{ml}$.

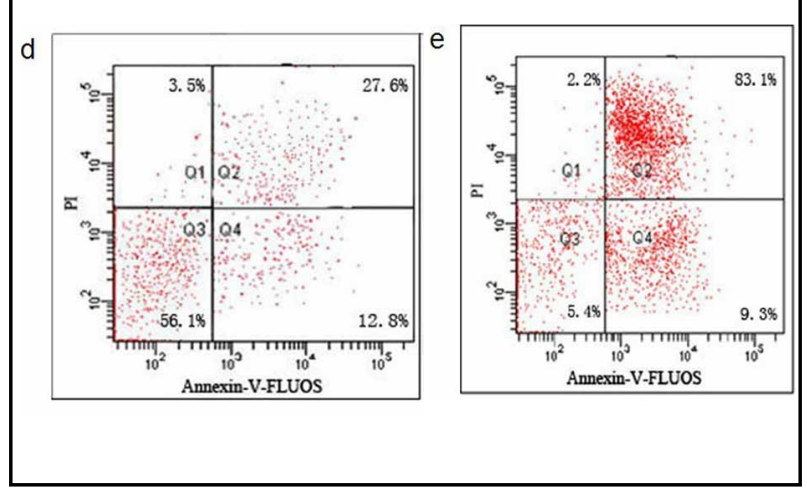

China), and the resultant cell suspension was centrifuged at $12000 \mathrm{r} / \mathrm{min}$ for $10 \mathrm{~min}$. The supernatant was removed and the amount of protein was quantified using the bicinchoninic acid (BCA) protein assay kit (Beyotime, China). The homogenized samples $(15 \mu \mathrm{g}$ crude proteins per lane) were subjected to electrophoresis on $12 \%(\mathrm{w} / \mathrm{v}$ ) poly-acrylamide-sodium dodecyl sulfate (SDS) gels under standard reducing conditions with precision multicolor protein marked (CWBIO, China), and then transferred to polyvinylidene difluoride membranes (Millipore, Bedford, MA, USA) at $2.5 \mathrm{~mA} / \mathrm{cm}^{2}$ for $45 \mathrm{~min}$. Then they were blocked with $5 \%(\mathrm{w} / \mathrm{v}$ ) BSA in PBS (blocking buffer) for $1 \mathrm{~h}$ at room temperature. Immunoblotting was carried out by incubating the membranes overnight at $4{ }^{\circ} \mathrm{C}$ with antibodies against Bcl2 and Bax (Santa,USA; 1:500 and 1:250 dilution) or GAPDH (Boster, China; 1:300 dilution). The membranes were washed three times with $1 \%$ (v/v) Tween-20 in PBS, then hybridized with horse radish peroxidase (HRP)-conjugated secondary goat anti-rabbit IgG antibody (Santa Cruz Biotechnology Inc., California, USA) diluted at 1:5000 for $2 \mathrm{~h}$. After three washes with $1 \%(\mathrm{v} / \mathrm{v})$ Tween-20 in PBS, the signals were detected as chemical luminescene by X-ray film with BeyoECL Plus kit (Beyotime, China).

\section{Measurement of cell growth by MTS assay}

Measurement of cell growth by MTS assay cumulus cells were counted in a hemocytometer, and the viability was determined by trypan blue dye exclusion, and then the cumulus cells were seeded in 96-well culture plates (Costar; Corning Inc., NY, USA) at a density of (3-5) $\times 10^{4}$ cells per well. The number of live cells at the end of the process of culture was determined with the MTS kit (Roche, China), as recommended by the manufacturer. The optical density at $490 \mathrm{~nm}$ (OD490) was determined using an enzyme-linked immunosorbent assay (ELISA) reader (Bio-TEK Instruments, USA).

\section{Statistical analyses}

Data were expressed as the SEM (mean \pm standard error of the mean) and analyzed by repeated measures analysis of variance (ANOVA), FCM, MTS and RT-qPCR data were also analyzed with Duncan's multiple-range tests using the SPSS13.0 software. $\mathrm{P}<0.05$ was considered to be significantly difference; $\mathrm{P}$ $<0.01$ was considered to be very significantly difference. 
Zhai et al.: Effects of BMP15 on Porcine Cumulus Cell Apoptosis

Table 2. Sequencing quality statistics for the cumulus cells treated with (BMP15) and without BMP15(N-BMP15)

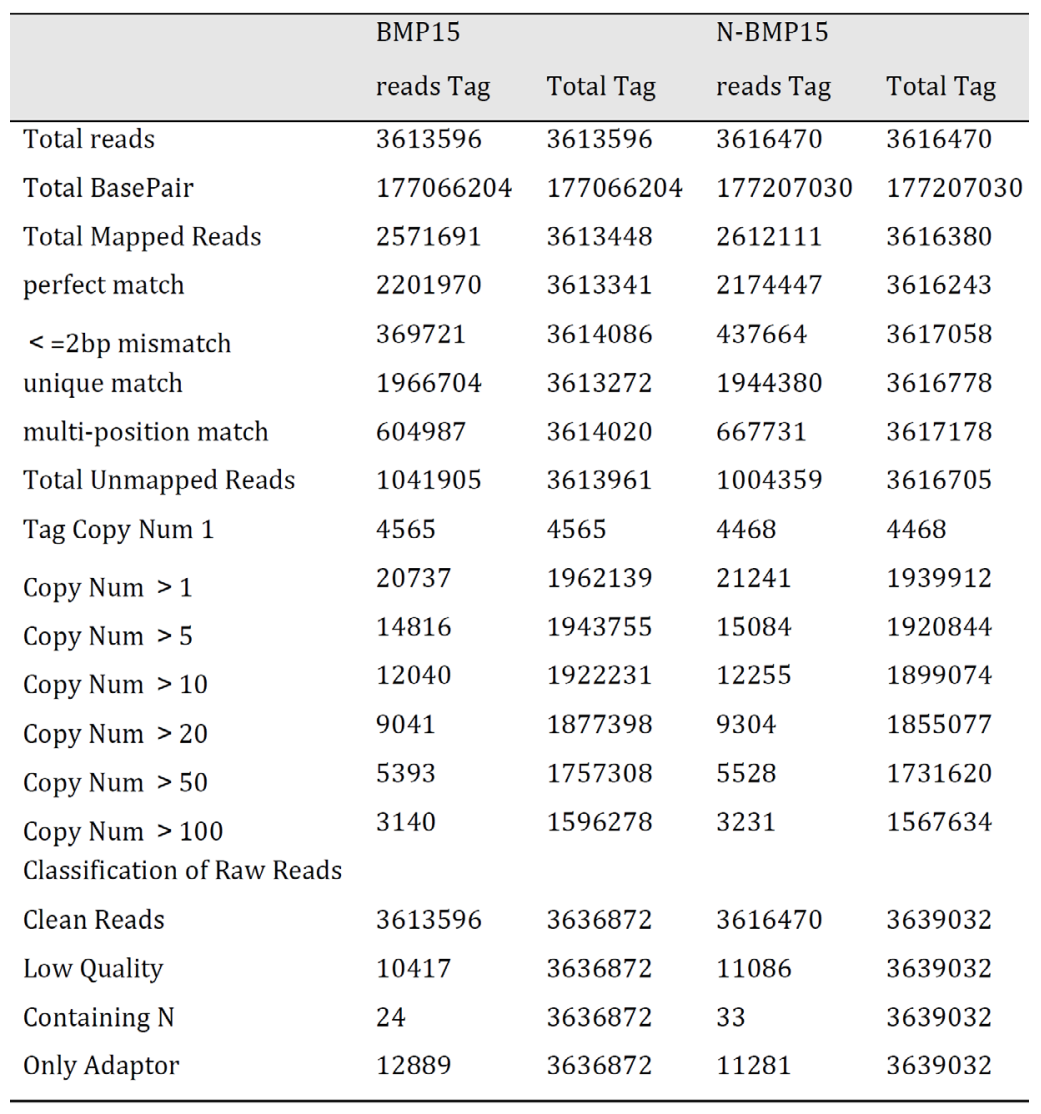

\section{Results}

\section{FCM analysis of apoptosis after BMP15 treatment}

After treatment with different doses of BMP15 for $96 \mathrm{~h}$, induction of apoptosis was analyzed using FCM. Apoptotic and necrotic cells were distinguished according to annexin V-FLOUS reactivity and propidium iodide (PI) exclusion. BMP15 $(0-200 \mathrm{ng} / \mathrm{ml})$ induced a dose-dependent decrease in apoptosis in cumulus cells (Fig. 1a-e). In particular, levels of cumulus cell apoptosis reduced significantly in cells treated with 50-200ng/ml of BMP15 (Fig. 1c-e). Fig. 1e shows that the death rate of cumulus cells which treated with $200 \mathrm{ng} / \mathrm{ml}$ of BMP15 increased significantly. It is suggested that cells cannot maintain normal growth at this concentration. BMP15 with $100 \mathrm{ng} / \mathrm{ml}$ was therefore chosen as the concentration for subsequent experiments.

\section{Analysis of differentially expressed gene (DEG) libraries}

In order to understand the regulation mechanism of the host cell's response to BMP15, the global gene expression profiles in cumulus cells was measured by using the Solexa highthroughput sequencing system based on tag transcriptome sequencing. DEG libraries were sequenced from two groups of porcine cumulus cells which treated with 100ng/ml BMP15 and untreated severally. The major sequencing results of the two libraries are summarized in Table 2. Approximately 3.6 million total sequence tags per library were obtained, among which there are 1.9 million distinct tag sequences. The raw reads were classified by filtered clean reads, low quality tags, the tags containing $\mathrm{N}$ and the sequences that consisted only of adapters before mapping the tag sequences to the reference sequences. We obtained approximately the same number of total clean sequence reads per library, with 3,613,596 
Fig. 2. Different gene expression in cumulus cells with BMP15 treatment. It was detected by Solexa high-throughput sequencing. A. The quantity of RNA used in Sole$\mathrm{xa}$, and the integrity of $18 \mathrm{~s}$ and 28 s rRNA was checked. B. The different expression of genes related to apoptosis. Expression of CCL2 / MCP-1, FBN1 and OXTR were significant difference than the other $(\mathrm{P}<0.05)$. C. CCL2 and FBN1 signals were detected in cumulus cells with the presence or absence of BMP15 by RT-qPCR. CCL2 was up-regulated and FBN1 was down-regulated in porcine cumulus cells with BMP15 treatment.

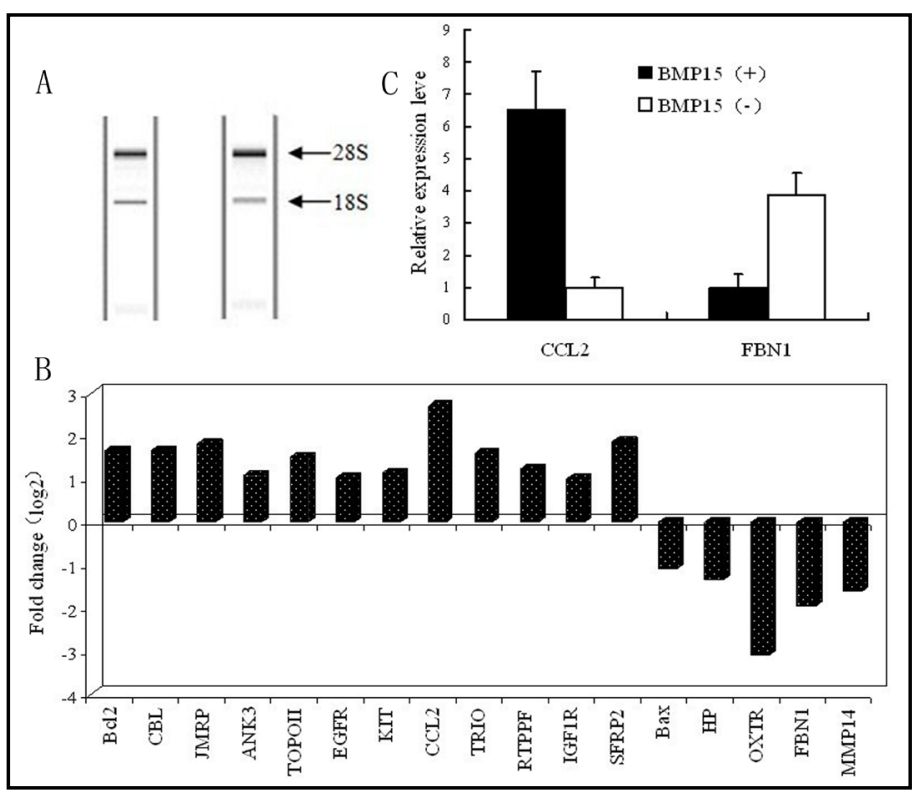

and 3,616,470 distinct clean read sequences. The tags with copy number $>1$ were then filtered and the data are shown in Table 2. The number of both total sequence tags and distinct sequence tags in N-BMP15 library is higher than the BMP15 libraries.

For tag mapping, total 2,571,691 mapped reads and 1,041,905 unmapped read sequences were obtained in the BMP15 treatment sample while total 2,612,111 mapped reads and 1,004,359 unmapped read sequences in the N-BMP15 treatment sample. Two mismatches in each alignment were allowed considering polymorphisms in the samples. Under these criteria, $54.43 \%$ and $53.76 \%$ of unique match tags mapped to the Unigene tag database, $60.94 \%$ and $60.13 \%$ of the perfect match tags mapped unambiguously to Unigene, $28.83 \%$ and $27.77 \%$ of the unmapped reads tags can not map to the database (Table 2).

Expression of CCL2 (MCP-1) and FBN1 was altered in cumulus cells after BMP15 treatment

The genes related to apoptosis are summarized in Fig. 2B. CCL2 (MCP-1), FBN1 and OXTR may be involved in apoptosis based on GO and Pathway analysis. The expression levels of CCL2 and FBN1 in cumulus cells were measured in the presence or absence of BMP15 by RT-qPCR. The results showed that CCL2 was upregulated and FBN1 was downregulated in porcine cumulus cells with BMP15 treatment (Fig. 2C). Moreover, because of previous research indicates that CCL2 suppresses apoptosis [14] and FBN1 induces apoptosis in vitro [15], the two genes were chosen for further research.

Validation of DEG data by RT-qPCR

To validate DEGs identified by Solexa sequencing, we randomly selected 11 genes from the significant DEGs. Five downregulated genes, i.e. epithelial chloride channel protein (CAV2), tubulin alpha-1C chain-like (alpha-1C), L1 cell adhesion molecule (L1CAM), secreted frizzled-related protein 2-like (SFRP2) and matrix metallopeptidase 14 (MMP14) and six upregulated genes, i.e. TGF- $\beta$ activated kinase $1 \mathrm{~b}$ (TAK1b), triple functional domain (PTPRF interacting) (TRIO), cytoskeleton associated protein 2 (CKAP2), bone morphogenetic protein receptor II (BMPR2), growth factor receptor (IGF1R) and topoisomerase II (TOPOII) were included. The data are presented as fold changes $(\log 2)$ in gene expression normalized to the $\beta$-actin gene and relative to the N-BMP15 sample. Pearson's correlation coefficient ( $\mathrm{r}$ ) showed that both the DEG and RT-qPCR data (pooling samples) were highly correlated, as the genes modulated by BMP15 had a high consistency (Fig. 3). It is confirmed from RT-qPCR analysis that the direction of change detected by DEG analysis. 
Fig. 3. Validation of the sequencing results by RT-qPCR. Histograms of the relative expression levels of BMP15+ / BMP15- (Cells with BMP15 treatment, BMP15+; Cells without BMP15 treatment, BMP15-).The $\mathrm{x}$-axis represents the mRNAs, and the $y$ -axis is the fold-change between the two samples (fold quantity values for RT-qPCR;

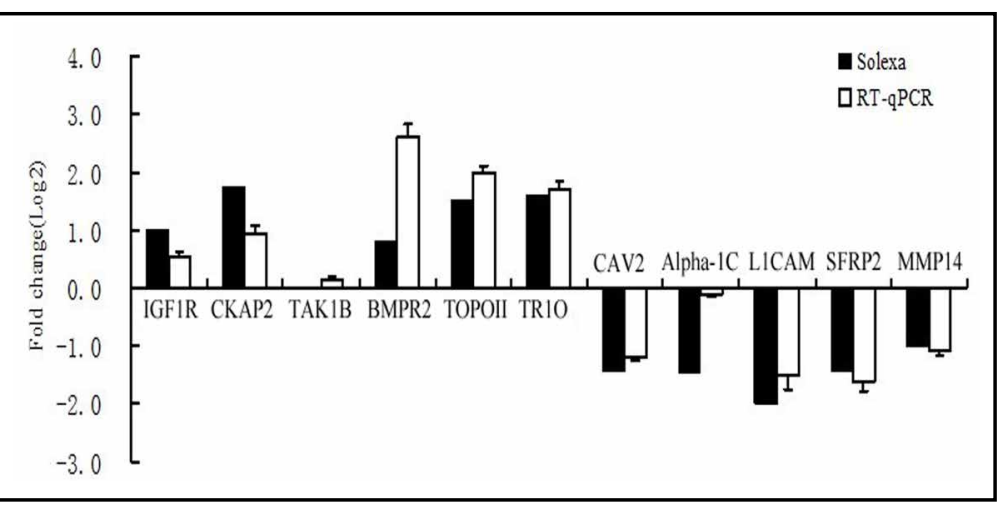
$\log$ (BMP15+ / BMP15-, 2) for sequencing).Three biological replicates were used, and the results were normalized to the expression level of $\beta$-actin.

Effect of siRNA against CCL2 on apoptosis and proliferation of cumulus cells

In order to study the function of CCL2 in the BMP15-mediated protection of cumulus cells from apoptosis, cumulus cell indicators were measured at the morphological, mRNA and protein levels. Flow cytometry experiments were used to describe the effects of adding BMP15 after the transfection of CCL2 siRNA on apoptosis rates in cumulus cells. Apoptotic cells were differentiated from viable or necrotic cells according to annexin V-FLUOS reactivity and PI exclusion. The results were shown in Fig. 4A. It can be seen that the cumulus cell apoptosis rate increased from $12.6 \%$ to $31.7 \%$ which the difference was significant $(\mathrm{P}<0.01)$ while CCL2 depletion after the addition of BMP15. However, the cumulus cell apoptosis rate increased from $29.2 \%$ to $31.7 \%$ which the difference was insignificant while CCL2 depletion before the addition of BMP15. It can be seen the effect of BMP15 is small without CCL2. These results indicated that BMP15 promotes cumulus cell apoptosis by silencing CCL2.

Quantitative fluorescence experiments were used to investigate the effects of adding BMP15 after the transfection of CCL2 siRNA on the levels of apoptotic gene mRNAs in cumulus cells. The results were shown in Fig. 4B. It can be seen that after the addition of BMP15, depletion of CCL2 led to a significant decrease in Bcl2 expression ( $P<0.01)$, a significant increase in Bax expression $(\mathrm{P}<0.01)$ and a significantly decreased ratio of the two $(\mathrm{P}<0.01)$. In addition, added BMP15 after CCL2 depletion did not lead to significant changes in the expression of Bax and Bcl2. It is suggested that BMP15 acts on cells via CCL2 and the protective effect of BMP15 against apoptosis was eliminated after depleting CCL2. These results indicated that BMP15 promotes cumulus cell apoptosis by silencing CCL2.

Western blotting experiments were used to study the effects of adding BMP15 after the transfection of CCL2 siRNA on the protein levels of cumulus cell apoptotic genes. Fig. 4C shows that after the addition of BMP15, CCL2 depletion led to a significantly decreased level of Bcl2 $(\mathrm{P}<0.01)$, significantly increased level of $\mathrm{Bax}(\mathrm{P}<0.05)$ and a significant reduction ratio of the two $(\mathrm{P}<0.01)$. In contrast, adding BMP15 after CCL2 depletion had little effect on the protein levels of Bax and Bcl2, suggesting that BMP15 can also act on the protein level to promote cumulus cell apoptosis by silencing the CCL2 gene.

Cumulus cell MTS (3-(4,5-dimethylthiazol-2-yl)-5-(3-carboxymethoxyphenyl)-2(4-sulfophenyl)-2H-tetrazolium) proliferation experiments were used to describe the effects of adding BMP15 after the transfection of CCL2 siRNA on cumulus cell proliferation. As shown in Fig. 4D, the growth states of cells showed no significant changes from $24 \mathrm{~h}$ to $72 \mathrm{~h}$ and the cell growth trend enhanced with an upward S-curve pattern after $72 \mathrm{~h}$. Cells were transfected with CCL2 siRNA after being cultured for $96 \mathrm{~h}$. At $24 \mathrm{~h}$ after transfection, that is to say the time point of $120 \mathrm{~h}$, the proliferation of cells in each group changed significantly. 


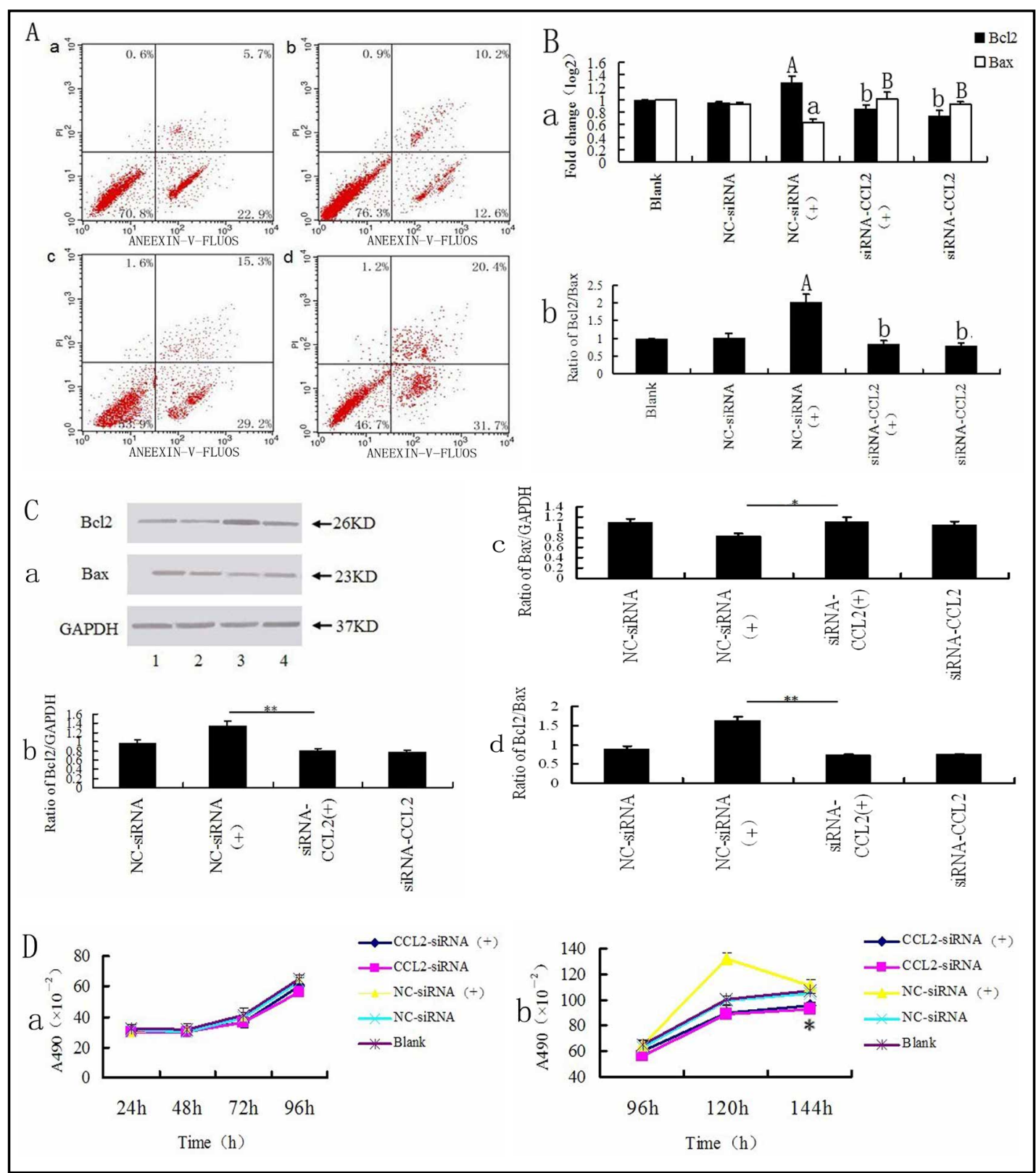

Fig. 4. SiRNA -CCL2 induces apoptosis and inhibits proliferation of cumulus cells in vitro. A. Flow cytometry was used to observe the apoptosis of cumulus cells by staining with annexinV-FLUOS/PI. a. NC-siRNA group; b. NC-siRNA(BMP15+) group; c. siRNA-CCL2 group; d. siRNA-CCL2(BMP15+)group; B. RT-qPCR analyses of Bcl-2 and Bax mRNA in porcine cumulus cells a. Relative Bcl-2 and Bax mRNA levels; $b$. Ratios of Bcl-2 to Bax. Bars indicate the mean \pm SEM. Different capital letters indicate high statistical difference $(P<0.01)$. Different lower case letters indicate statistical difference $(\mathrm{P}<0.05)$.This assay was done quadruplicate. Values represent means \pm standard deviations $(n=4)$ and were determined using the Student's t-test. C. Western blot analyses of Bcl-2 and Bax proteins in porcine cumulus cells. 1. siRNA-CCL2(BMP15+)group 2. siRNACCL2 group; 3. NC-siRNA(BMP15+) group; 4. NC-siRNA group; Optical density of the bands was determined by Image J software and normalized using internal reference gene (GAPDH).b. Relative Bcl-2 protein levels; c. relative Bax protein levels; d. ratios of Bcl-2 to Bax. Bars indicate the mean \pm SEM of three experiments. ${ }^{* *} \mathrm{P}<0.01$ indicate high statistical difference, ${ }^{*} \mathrm{P}<0.05$ indicate statistical difference. D. Cell viability analyses of the growth curve in porcine cumulus cells by MTS method. This assay was performed in triplicate. Dosedependent inhibition of cell growth could be observed after $96 \mathrm{~h}$ when the cells were transfected. a. The $96 \mathrm{~h}$ before cells transfection; b. The $48 \mathrm{~h}$ after cells transfection. $\left({ }^{*} \mathrm{P}<0.05\right.$, ANOVA analysis). 


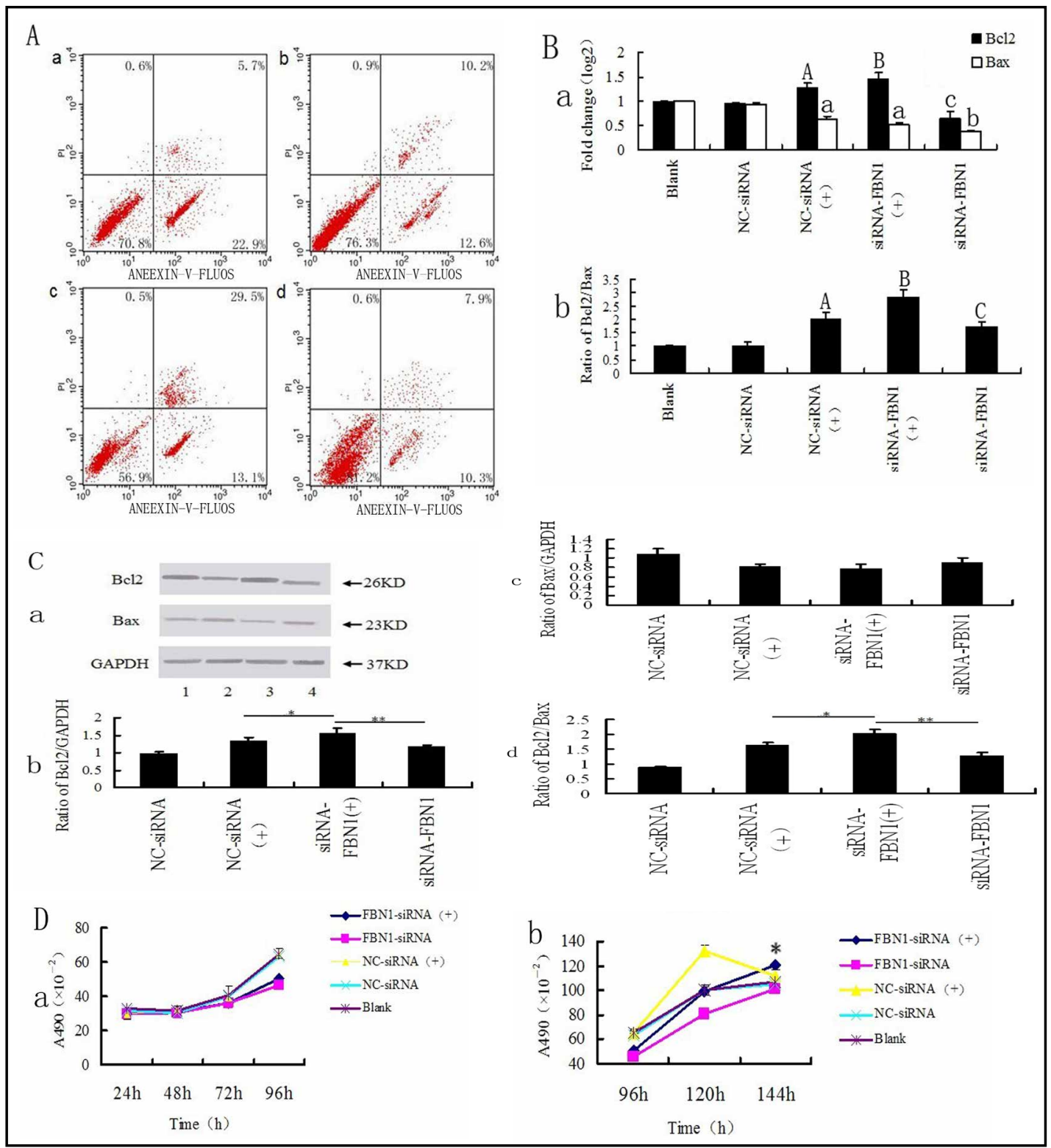

Fig. 5. SiRNA -FBN1 induces apoptosis and inhibits proliferation of cumulus cells in vitro. A. Flow cytometry was used to observe the apoptosis of cumulus cells by staining with annexinV-FLUOS/PI. Percentage values represent the population of Annexin $\mathrm{V}+$ /PI-cells undergoing apoptosis.a. NC-siRNA group; $b$. NC-siRNA(BMP15+) group; c. siRNA- FBN1 group; d. siRNA-FBN1 (BMP15+)group; B. RT-qPCR analyses of Bcl-2 and Bax mRNA in porcine cumulus cells a. Relative Bcl-2 and Bax mRNA levels; $\mathrm{b}$. Ratios of Bcl-2 to Bax. Bars indicate the mean \pm SEM. Different capital letters indicate high statistical difference $(P<0.01)$. Different lower case letters indicate statistical difference $(\mathrm{P}<0.05)$.This assay was done quadruplicate. Values represent means \pm standard deviations $(n=4)$ and were determined using the Student's t-test. C. Western blot analyses of Bcl-2 and Bax proteins in porcine cumulus cells. 1. NC-siRNA(BMP15+) group; 2.NC-siRNA group; 3. siRNA-FBN1(BMP15+)group; 4.siRNA-FBN1 group. Optical density of the bands was determined by Image J software and normalized using internal reference gene (GAPDH). b. Relative Bcl-2 protein levels; c. relative Bax protein levels; d. ratios of Bcl-2 to Bax. Bars indicate the mean \pm SEM. ${ }^{* *} \mathrm{P}<0.01$ indicate high statistical difference, ${ }^{*} \mathrm{P}<0.05$ indicate statistical difference.D. Cell viability analyses of the growth curve in porcine cumulus cells by MTS method after the cells were transfected. This assay was performed in triplicate. Different inhibition of cell growth could be observed after $96 \mathrm{~h}$ when the cells were transfected. a. The $96 \mathrm{~h}$ before cells transfection; b. The $48 \mathrm{~h}$ after cells transfection $(\mathrm{P}<0.05$, ANOVA analysis). 
Furthermore, at $48 \mathrm{~h}$ after transfection, that is to say the time point of $144 \mathrm{~h}$ (the time point showing the maximum effect of siRNA transfection), the absorbance value of the group transfected with CCL2 siRNA after BMP15 addition was significantly decreased compared to the untransfected group ( $\mathrm{P}<0.05)$. In contrast, after the depletion of CCL2, the addition of BMP15 had no significant effects on the absorbance values of cells. This result indicates that CCL2 gene silencing prevents BMP15 from promoting cumulus cell proliferation.

Effect of FBN1 siRNA on the apoptosis and proliferation of cumulus cells

In order to study the function of FBN1 in the BMP15-mediated protection of cumulus cells from apoptosis, cumulus cell indicators were measured at the morphological, mRNA and protein levels. FCM experiments were carried out to investigate the increased effects of BMP15 after FBN1 siRNA transfection on the apoptosis rate of cumulus cells. The results in Fig. 5A show that after adding BMP15, depletion of FBN1 reduced the apoptosis rate of cumulus cells from $12.6 \%$ to $10.3 \%$ and the difference was significant $(\mathrm{P}<0.05)$. Moreover, adding BMP15 after FBN1 depletion reduced the apoptosis rate of cumulus cells from 13.1\% to $10.3 \%$ which was significant $(P<0.05)$. These results suggest that silencing FBN1 gene effectively would inhibit cumulus cell apoptosis while BMP15 existence. In addition, the results also indicate BMP15 and other factors have synergistic effects based on the fact that the BMP15-mediated inhibition of apoptosis was more significant in cells after FBN1 was depleted.

Furthermore, according to the results from quantitative fluorescence experiments, it is explored the effects of adding BMP15 after the transfection of FBN1 siRNA on the mRNA levels of apoptotic genes in cumulus cells. Fig. 5B shows that the depletion of FBN1 led to a significant increase in Bcl2 expression $(\mathrm{P}<0.05)$ but no significant changes in Bax expression after adding BMP15, and the ratio of the two was significantly increased $(\mathrm{P}<0.01)$. In contrast, after FBN1 depletion, the addition of BMP15 led to a significant increase in the expression levels of both $\mathrm{Bcl} 2$ and $\mathrm{Bax}(\mathrm{P}<0.01$ and $\mathrm{P}<0.05$, respectively), and the ratio of the two was significantly increased $(\mathrm{P}<0.01)$. These results suggest that BMP15 suppresses cumulus cell apoptosis by silencing FBN1 and BMP15 also participates in the regulation of other upregulated genes to further protect against cumulus cell apoptosis.

Western blotting experiments were performed to determine the effects of adding BMP15 after the transfection of FBN1 siRNA on the protein levels of cumulus cell apoptotic genes. Fig. 5C shows that after adding BMP15, FBN1 depletion led to a significant increase in $\mathrm{Bcl} 2$ protein level $(\mathrm{P}<0.05)$, whereas the level of Bax has no significant changes, and the ratio of the two significantly increased $(\mathrm{P}<0.05)$. In contrast, adding BMP15 after CCL2 depletion led to a significant increase in Bcl2 expression $(\mathrm{P}<0.01)$, whereas the level of the Bax protein has no significantly change, and the ratio between the two was significantly increased $(\mathrm{P}<0.01)$, suggesting that BMP15 can act on the protein level to inhibit cumulus cell apoptosis by silencing the FBN1 gene.

Using MTS proliferation experiments in cumulus cells, we described the effects of adding BMP15 after the transfection of FBN1 siRNA on cumulus cell proliferation. As shown in Fig. 5D, the growth states of cells in each group showed no significant changes from 24 $\mathrm{h}$ to $72 \mathrm{~h}$, and after $72 \mathrm{~h}$, the cell growth trend showing an upward S-curve pattern and the number of cells increased significantly,. Cells were transfected with FBN1 siRNA after being cultured for $96 \mathrm{~h}$. At $24 \mathrm{~h}$ after transfection, that is to say the time point of $120 \mathrm{~h}$, the proliferation of cells in each group changed significantly. At $48 \mathrm{~h}$ after transfection, that is to say the time point of $144 \mathrm{~h}$ (the time point showing the maximum effect of transfection), the absorbance values of the group transfected with FBN1 siRNA after adding BMP15 were significantly increased compared with the untransfected group $(\mathrm{P}<0.05)$. In contrast, after depleting FBN1, the addition of BMP15 significantly increased the absorbance values of cells $(\mathrm{P}<0.05)$. This result indicates that silencing the FBN1 gene facilitates the promotion of cumulus cell proliferation by BMP15. 


\section{Discussion}

Follicular atresia is a complex and cumbersome process. It involves a variety of signaling pathways and regulatory factors. Some of these factors regulate follicular atresia by controlling the fate of granulosa cells. Oocyte-secreted factors have been shown to play critical roles in the regulation of follicular cell functions [16]. BMP15 is one of the intrinsic factors that play essential roles in promoting granulosa cell survival. Granulosa cells are divided into two types: mural granulosa cells (MGC) and cumulus cells. Cumulus cells are specialized cells that are actually derived from the granulosa cells that surround and nourish the oocyte during maturation [17]. Cumulus cell apoptosis plays a critical role in the growth and development of the oocyte. BMP15 is specifically secreted by the oocyte within the mammalian ovary and is important for maintenance of the tertiary structure of COC. However, the effect of BMP15 on the apoptotic mechanism of cumulus cells has not been reported. Previous studies have indicated that BMP15 may protect bovine cumulus cell apoptosis [10]. Accordingly, this paper also proves that BMP15 may protect the porcine cumulus cell apoptosis. Also, BMP15 treatment can significantly influence the expression of CCL2 and FBN1, and the protective effects of BMP15 against apoptosis were regulated by changing the expression of CCL2 and FBN1. To our knowledge, this is the first evidence demonstrating the roles of CCL2 and FBN1 in relation to BMP15 in female reproduction and, more specifically, in follicular atresia.

In this study, FCM was used to measure the apoptosis of cumulus cells treated with different concentrations of BMP15. The results showed that the apoptosis rate of cumulus cells treated with BMP15 changed in a dose-dependent manner, which is in agreement with a previous study. The reduced level of cumulus cell apoptosis is dependent on the presence of BMP15 [10]. We found that BMP15 effectively prevented the apoptosis of cumulus cells in vitro at a concentration of $100 \mathrm{ng} / \mathrm{ml}$, which was used for further studies. However, concentrations of $200 \mathrm{ng} / \mathrm{ml} \mathrm{BMP15}$ caused a very high rate of cell death, and cumulus cells did not grow normally. The reason might be the high concentrations of exogenous additives affected normal cell growth and may have even caused death.

BMP15 may activate different signaling pathways during follicle development [18]. Prosurvival and pro-apoptotic molecules are involved in ovarian apoptosis [19], and a delicate balance between them determines the final destiny of follicular cells [20]. Our results suggest that BMP15 regulates many genes involved in the regulation of the pro-survival and pro-apoptotic gene balance in cumulus cell apoptosis. It has been demonstrated that the FAS pathway ameliorates disease phenotypes in FBN-1-mutant systemic sclerosis (SS) and dextran-sulfate-sodium-induced experimental colitis. FAS-regulated CCL2 secretion by bone marrow mesenchymal stem cells (BMMSCs) recruits T cells for FASL-mediated apoptosis [14]. It is known that FAS and FASL are key factors for cumulus cell apoptosis, and the close connection between the two genes in cumulus cell apoptosis is clear. In this study, hundreds of different genes were pretested, tens of which were involved in apoptosis, including proapoptotic genes (such as FBN1, Bax and OXTR) and anti-apoptotic genes (such as CCL2, $\mathrm{Bcl} 2$, kit and EGFR). Among them, we found that after BMP15 treatment, the changes in the expression levels of CCL2 and FBN1 were more significant. Therefore, we focused on these two genes for the subsequent in-depth study. Our results suggest the involvement of CCL2 upregulation and FBN1 downregulation in the regulation of cumulus cell apoptosis, providing a new possible mechanism for the action of BMP15.

MCP-1, also known as CCL2, was the first identified member of the chemokine subfamily and is secreted by a variety of immune and non-immune cell types, such as monocytes, epithelial cells, smooth muscle cells and fibroblasts [21]. The involvement of chemokines in ovarian function is becoming more evident as research in the area of reproduction progresses. CCL2 is directly or indirectly related to follicular development and atresia, ovulation, steroidogenesis and corpus luteum (CL) function [22]. Bcl2 and Bax are two markers for apoptosis; Bcl2 is an anti-apoptotic gene, and Bax is a pro-apoptotic gene. The ratio between the two determines the fate of a cell, survival or death [23]. Therefore, in the 
present study, we utilized these two apoptotic marker genes to detect apoptosis at the mRNA and protein levels. This study analyzed the underlying mechanism by which CCL2 protects follicles from atresia, that is, its effects on cumulus cell apoptosis, in the presence of BMP15. After BMP15 addition, the depletion of CCL2 promoted cumulus cell apoptosis; however, adding BMP15 after CCL2 was depleted had no obvious effect. These results suggest that the pro-apoptotic effect observed in the former experiment may have been because BMP15 protected the cumulus cells from apoptosis via CCL2, and after silencing CCL2, the protective effect of BMP15 was lost. In the latter experiment, due to the lack of the CCL2 gene, BMP15 lost the ability to protect cumulus cells from apoptosis. Hence, there may be a necessary connection between BMP15 and CCL2 enabling the two to regulate cumulus cell apoptosis by regulating each other. There are many reports showing that CCL2 influences apoptosis by regulating intracellular factors. CCL2 cytoprotection and chemotaxis may occur through distinct signaling mechanisms. CCL2 protects cultured mouse neonatal cardiac myocytes from hypoxia-induced cell death [24]. CCL2 protection from tat-induced apoptosis in neurons correlates with a reduction in uptake and changes in the intracellular distribution of tat [25]. CCL2 also inhibits the T cell apoptotic program induced by growth factor deprivation [26] and blocks the constitutive apoptosis of normal neutrophils through CCR2 [27]. These results agree with our data and substantiated our results. CCL2 may play an anti-apoptotic role in the above cell types when cells sense external stimulation. Similar to the above cell types, in the cumulus cells used in this study, CCL2 acts as a typical chemokine and facilitates the anti-apoptotic effect of BMP15 through changes in its own expression only in the presence of BMP15. A previous report revealed that FAS-regulated CCL2 secretion by BMMSCs recruited T cells for FASL-mediated apoptosis [14]. FAS/FASL is the primary ligand system affecting the apoptosis of granular cells, and CCL2 interacts with this system, thus regulating apoptosis. The protective effect of BMP15 against apoptosis that is discussed in this paper may also be achieved by stimulating the FAS/FASL system by regulating CCL2, thus protecting cumulus cells from apoptosis. Therefore, this study identified potential regulatory factors by which BMP15 protects cumulus cells from apoptosis.

FBN1, a 350-kD glycoprotein, is the main component of microfibrils in the extracellular matrix [28]. FBN1 plays a crucial role in fibrosis by regulating TGF- $\beta$ signaling in the extracellular matrix [29]. In the ovary, FBN1 expression and localization affect TGF- $\beta$ bioavailability [30]. This study analyzed the underlying mechanism by which FBN1 stimulates follicular atresia, that is, its effects on cumulus cell apoptosis, in the presence of BMP15. In the presence of BMP15, FBN1 depletion protected cumulus cells from apoptosis; similarly, when FBN1 was depleted, the addition of BMP15 played a protective role for cumulus cells. However, the effect in the latter experiment was more significant than that in the former experiment. This may be because regardless of the silencing of FBN1 gene, the presence of BMP15 upregulated the intracellular level of CCL2, which in turn enhanced the protective effect of BMP15 against cumulus cell apoptosis, thus leading to a further enhancement of the protective effect of BMP15 against cumulus cell apoptosis under these conditions. This conclusion suggests that the connections between BMP14 and CCL2 and between BMP15 and FBN1 can regulate cumulus cell apoptosis. Although there are no previous reports in the literature describing a direct relationship between FBN1 and apoptosis, changes in FBN1 genotype have been shown to affect T cell apoptosis [14]. Here, we showed that BMP15 downregulated the FBN1 gene, with the result that FBN1 was no longer involved in the regulation of apoptotic pathways. In other words, downregulation of the FBN1 gene enhanced the protective effect of BMP15 on cumulus cells. A previous study showed that mice deficient in FBN1 have marked dysregulation of TGF- $\beta$ activation and signaling, resulting in apoptosis in the developing lung [15]. FBN1 deficiency activates and leads to abnormalities in TGF- $\beta$ signaling, suggesting that FBN1 may regulate the activity of the TGF- $\beta$ family. BMP15 is a typical member of the TGF- $\beta$ family. It is likely that there is a connection between BMP15 and FBN1 that regulates host cell apoptosis and proliferation. It has been reported in the literature that FBN1 deficiency causes apoptosis in the lung, which differs from the conclusion derived from the present study. A possible reason may 
be that different mechanisms are involved in gene regulation in different tissues and cells or that variations in gene function in different tissues were caused by the differences in the internal environments of the organisms in the in vivo and in vitro studies. The induction of apoptosis and FBN1 expression was observed in human dermal endothelial cells treated with scleroderma sera containing anti-endothelial cell antibodies [31], indicating that certain stimuli can change the FBN1 phenotype or expression level. This conclusion supports the results of the present study regarding FBN1 expression in apoptotic cells. Here, we showed that BMP15 promotes the apoptosis of cumulus cells by reducing the expression level of FBN1.

In conclusion, the present study indicates that BMP15 inhibits cumulus cell apoptosis through a different mechanism, such as CCL/FBN1. More details regarding the regulatory pathways involving BMP15/CCL2 and BMP15/FBN1 need to be verified by further studies. The present data provide new research avenues for the role of BMP15 in the apoptosis pathway. Many aspects of the effects of BMP15 in mammalian reproduction remain to be elucidated. In addition, because the pig has high homology with humans, the function of BMP15 in pigs can be used to model the mechanisms of cumulus cell apoptosis during human reproduction.

\section{Acknowledgments}

Research was supported by the National Natural Science Foundation of China (31030058).

\section{References}

1 Tilly J L: Apoptosis and ovarian function. Rev Reprod 1996;1: 62-172.

- Kerr JF, Wyllie AH, Currie AR: Apoptosis: a basic biological phenomenon with wide-ranging implication in tissue kinetics. Br J Cancer 1972;26:239-257.

-3 Hsu SY, Hsueh AJ: Hormonal regulation of apoptosis an ovarian perspective. Trends Endocrinol Metab 1997;8:207-213.

-4 Galloway SM, McNatty KP, Cambridge LM, Laitinen MP, Juengel JL, Jokiranta TS, McLaren RJ, Luiro K, Dodds KG, Montgomery GW, Beattie AE, Davis GH, Ritvos O: Mutations in an oocyte-derived growth factor gene (BMP15) cause increased ovulation rate and infertility in a dosage-sensitive manner. Nat Genet 2000;25:279-283.

-5 Aaltonen J, Laitinen MP, Vuojolainen K, Jaatinen R, Horelli-Kuitunen N, Seppä L, Louhio H, Tuuri T, Sjöberg J, Bützow R, Hovata O, Dale L, Ritvos O: Human growth differentiation factor 9 (GDF-9) and its novel homolog GDF-9B are expressed in oocytes during early folliculogenesis. J Clin Endocrinol Metab 1999;84:2744-2750.

6 Shimasaki S, Moore RK, Otsuka F, Erickson GF: The bone morphogenetic protein system in mammalian reproduction. Endocr Rev 2004;25:72-101.

$>7$ Otsuka F, Yamamoto S, Erickson GF, Shimasaki S: Bone morphogenetic protein-15 inhibits folliclestimulating hormone (FSH) action by suppressing FSH receptor expression. J Biol Chem 2001;276:1138711392.

-8 Otsuka F, Shimasaki S: A novel function of bone morphogenetic protein-15 in the pituitary: selective synthesis and secretion of FSH by gonadotropes. Endocrinology 2002;143:4938-4941.

-9 Pangas SA, Jorgez CJ, Matzuk MM: Growth differentiation factor 9 regulates expression of the bone morphogenetic protein antagonist gremlin. J Biol Chem 2004;279:32281-32286.

$\rightarrow 10$ Hussein TS, Froiland DA, Amato F, Thompson JG, Gilchrist RB: Oocytes prevent cumulus cells apoptosis by maintaining a morphogenic paracrine gradient of bone morphogenetic proteins. J Cell Sci 2005;118:52575268. 
11 Wajant H, Scheurich P: Tumor necrosis factor receptor-associated factor (TRAF) 2 and its role in TNF signaling. Int J Biochem Cell Biol 2001;33:19-32.

12 Manabe N, Matsuda-Minehata F, Goto Y, Maeda A, Cheng Y, Nakagawa S, Inoue N, Wongpanit K, Jin H, Gonda H, Li J: Role of cell death ligand and receptor system on regulation of follicular atresia in pig ovaries. Reprod Domest Anim 2008;79:1-10.

13 Manabe N, Goto Y, Matsuda-Minehata F, Inoue N, Maeda A, Sakamaki K, Miyano T: Reulation Mechanism of Selective Atresia in Porcine Follicles: Regulation of Granulosa Cell Apoptosis during Atresia. J Reprod Dev 2004;50:493-514.

14 Akiyama K, Chen C, Wang D, Xu X, Qu C, Yamaza T, Cai T, Chen W, Sun L, Shi S: Mesenchymal-StemCell-Induced Immuno regulation Involves FAS-Ligand/FAS-Mediated T Cell Apoptosis. Cell Stem Cell 2012;10:544-555.

15 Neptune ER, Frischmeyer PA, Arking DE, Myers L, Bunton TE, Gayraud B, Ramirez F, Sakai LY, Dietz HC: Dysregulation of TGF- $\beta$ activation contributes to pathogenesis in Marfan syndrome. Nat Genet 2003;33:407-411.

16 Gilchrist RB, Ritter LJ, Armstrong DT: Oocyte-somatic cell interactions during follicle development in mammals. Anim Reprod Sci 2004;82-83:431-446.

17 Polzikov M, Yakovenko S, Voznesenskaya J, Troshina M, Zatsepina O: Overexpression of ribosomal RNA in cumulus cells of patients with polycystic ovary syndrome. J Assist Reprod Genet 2012;29:1141-1145.

18 Moore R K, Otsuka F, Shimasaki S: Molecular basis of bone morphogenetic protein-15 signaling in granulosa cells. J Biol Chem 2003;278:304-310.

19 Matsuda-Minehata F, Inoue N, Goto Y, Manabe N: The regulation of ovarian granulosa cell death by pro- and anti-apoptotic molecules. J Reprod Dev 2006;52:695-705.

20 Hussein MR: Apoptosis in the ovary: molecular mechanisms. Hum Reprod Update 2005;11:162-177.

21 Niu J and Kolattukudy PE: Role of MCP-1 in cardiovascular disease: molecular mechanisms and clinical implications. Clin Sci (Lond) 2009;117:95-109.

22 Kayisli UA, Mahutte NG, Arici A: Uterine Chemokines in Reproductive Physiology and Pathology. Am J Reprod Immunol 2002;47:213-221.

23 Oltvai Z N, Milliman C L, Korsmeyer S J: Bcl-2 heterodimerizes in vivo with a conserved homolog, Bax, that accelerates programmed cell death. Cell 1993;74:609-619.

-24 Tarzami ST, Calderon TM, Deguzman A, Lopez L, Kitsis RN, Berman JW: MCP-1/CCL2 protects cardiac myocytes from hypoxia-induced apoptosis by a Gai-independent pathway. Biochem Biophys Res Commun 2005;335:1008-1016.

25 Eugenin EA, D'Aversa TG, Lopez L, Calderon TM, Berman JW: MCP-1 (CCL2) protects human neurons and astrocytes from NMDA or HIV-tat-induced apoptosis. J Neurochem 2003;85:1299-1311.

26 Diaz-Guerra E, Vernal R, del Prete MJ, Silva A, Garcia-Sanz JA: CCL2 Inhibits the Apoptosis Program Induced by Growth Factor Deprivation, Rescuing Functional T Cells. J Immunol 2007;179:7352-7357.

-27 Yang EJ, Choi E, Ko J, Kim DH, Lee JS, Kim IS: Differential effect of CCL2 on constitutive neutrophil apoptosis between normal and asthmatic subjects. J Cell Physiol 2012;227:2567-2577.

-28 Dietz HC, Cutting GR, Pyeritz RE, Maslen CL, Sakai LY, Corson GM, Puffenberger EG, Hamosh A, Nanthakumar EJ, Curristin SM, et al: Marfan syndrome caused by a recurrent de novo missense mutation in the fibrillin gene. Nature 1991;352:337-339.

29 Neptune ER, Frischmeyer PA, Arking DE, Myers L, Bunton TE, Gayraud B, Ramirez F, Sakai LY, Dietz HC: Dysregulation of TGF $\beta$ activation contributes to pathogenesis in Marfan syndrome. Nat Genet 2003;33:407-411.

30 Prodoehl MJ, Irving-Rodgers HF, Bonner WM, Sullivan TM, Micke GC, Gibson MA, Perry VE, Rodgers RJ: Fibrillins and latent TGF $\beta$ binding proteins in bovine ovaries of offspring following high or low protein diets during pregnancy of dams. Mol Cell Endocrinol 2009;307:133-141.

-31 Ahmed SS, Tan FK, Arnett FC, Jin L, Geng YJ: Induction of Apoptosis and Fibrillin 1 Expression in Human Dermal Endothelial Cells by Scleroderma Sera Containing Anti-Endothelial Cell Antibodies. Arthritis Rheum 2006;54:2250-2262. 